\title{
Modeling of asymmetric membrane formation by a combination of dry/wet phase inversion processes
}

\author{
Sacide Alsoy Altinkaya \\ Department of Chemical Engineering, Izmir Institute of Technology, Gulbahce Koyu, 35437 Urla-İzmir, Turkey \\ email: sacidealsoy@iyte.edu.tr
}

Received 22 October 2005; accepted 4 March 2006

\section{Introduction}

The invention of asymmetric polymer membranes by Loeb and Sourirajan has caused an enormous growth in the field of membrane science and technology. Application areas range from chemical, biomedical to biotechnology industries. Asymmetric membranes are mostly fabricated by a process called phase inversion which can be achieved through four principal methods: immersion precipitation (wet phase inversion), vapor-induced phase separation, thermally induced phase separation and dry-casting. In these techniques, an initially homogeneous polymer solution becomes thermodynamically unstable and phase separates into polymerlean and polymer-rich phases. Structure of these types of membranes is greatly influenced by even slight change in membrane preparation conditions. Consequently, there has been considerable motivation for developing mathematical models which allow to eliminate extensive trial and error experimentation and optimize membrane preparation conditions.

Most of the modeling and experimental studies in the literature has focused on wet-cast and thermal-cast processes. There are few models for nonsolvent vapor-induced phase separation and dry-cast processes. On the other hand, an analysis of dry/wet phase inversion process is quite limited [1]. Pinnau and Koros [2] have shown that asymmetric membranes with ultrathin and defect free skin layers can only be manufactured by dry/wet phase inversion processes. A number of other studies have reported that membrane structures and performances can be significantly influenced by evaporation times on the order of few seconds before wet-casting step $[3,4]$. These observations clearly indicate that a mathematical model for combined dry/wet phase inversion process needs to be developed and applied to industrial practice.

\section{Theory}

The polymeric membrane formation by phase inversion is a complicated process due to phase seperation, simultaneous heat and mass transfer, complex thermodynamic and transport properties of polymer solutions. Modeling of combined dry/ wet phase inversion process introduces additional complexity due to the presence of a nonuniform concentration profile in the film at the beginning of the quenching (wet phase inversion) period. In this work, a fully predictive mathematical model is 
developed to describe the mechanisms of membrane formation by a combination of dry/wet phase inversion processes. Model equations consist of coupled heat and mass transfer equations for both periods, appropriate boundary conditions especially at polymer-gas and polymer-bath interfaces and an expression which determines the time dependence of boundary positions. A key element of the model is incorporation of multicomponent diffusivities. A robust algorithm developed previously by our group was used to construct the ternary phase diagram [5]. The algorithm utilizes Flory-Huggins thermodynamic theory with constant interaction parameters. The model is applied to cellulose acetate/acetone/water system which is commonly used for asymmetric membrane formation.

\section{Conclusion}

Results will illustrate how model predictions can provide information about the structure of the membrane. The effects of evaporation time, composition of the casting solution, relative humidity in air and initial thickness of the casting solution on the structure of the membrane will be discussed. In addition, the importance of diffusion formalism on the predictions will be evaluated.

\section{References}

[1] C.S. Tsay and A.J. McHugh, The combined effects of evaporation and quench steps on asymmetric membrane formation by phase inversion, J. Polym. Sci.: Part B: Polym. Phys., 29 (1991) 1261-1271.

[2] I. Pinnau and W. Koros, Structures and gas separation properties of asymmetric polysulfone membranes made by dry, wet, and dry/wet phase inversion, J. Appl. Polym. Sci., 43 (1991) 1491-1502.

[3] U. Rosenthal, J. Nechushtan, A. Kedem, D. Lancet and M.A. Frommer, An apparatus for studying the mechanism of membrane formation, Desalination, 9 (1971) 193-200.

[4] K.K. Sirkar, N.K. Agarwal and G.P. Rangaiah, The effect of short air exposure periods on the performance of cellulose acetate membranes from casting solutions with high cellulose acetate content, J. Appl. Polym. Sci., 22 (1978) 1919-1944.

[5] S.A. Altinkaya and B. Ozbas, Modeling of asymmetric membrane formation by dry casting method, J. Membr. Sci., 230 (2004) 71-89. 